\title{
Problemas gastrointestinales, nutricionales, endocrinológicos y de microbiota en el trastorno del espectro autista
}

\author{
Gastrointestinal, nutritional, endocrine, and microbiota \\ conditions in autism spectrum disorder
}

\author{
Dr. Nicolás Loyacono ${ }^{a, b}$, Lic. en Nutrición María L. Sanz ${ }^{b}$ Dra. María D. Gerbib,c, \\ Dr. Luis M. Martínez ${ }^{b}$, Dra. en Química María L. Ferreira ${ }^{b}$ Dr. Roberto Iermolia,b
}

\section{RESUMEN}

Los problemas médicos gastrointestinales, nutricionales, metabólicos, endocrinológicos y de microbiota en los pacientes pediátricos con diagnóstico de trastorno del espectro autista (TEA) son parte de los problemas médicos concomitantes al diagnóstico. La prevalencia alcanza a más del $91 \%$ en el caso de los problemas gastrointestinales, hasta el $89 \%$ para los nutricionales y metabólicos, más del $50 \%$ de disfunción tiroidea y hasta el $100 \%$ para los relacionados con la microbiota.

Es urgente actualizar la práctica médica para incluir la evaluación, testeo, diagnóstico y tratamiento de estos problemas médicos concomitantes al diagnóstico de TEA en la población pediátrica, adolescente y adulta. El tratamientoriguroso de dichos problemas genera cambios positivos en la calidad de vida y en la sintomatología bajo la cual el TEA se diagnostica en muchos casos. Debe basarse en evidencia científica de alta calidad, con control y cuidado médico adecuado.

Palabras clave: trastorno del espectro autista, enfermedades gastrointestinales, nutrición, microbiota, enfermedades del sistema endócrino.

http: / / dx.doi.org/10.5546/ aap.2020.e271

Texto completo en inglés:

http:/ / dx.doi.org/10.5546/ aap.2020.eng.e271

Conflicto de intereses: La Dra. en Química María Luján Ferreira, madre de un joven al que le diagnosticaron trastorno del espectro autista en 2003, participó como asesora científica $a d$ honórem del Hospital de Clínicas José de San Martín según la Resolución 3070/2015 del Directorio del Consejo Nacional de Investigaciones Científicas y Técnicas (CONICET) desde el 1/6/2015 hasta el $30 / 6 / 2019$.

Recibido: 4-7-2019

Aceptado: 17-12-2019
Cómo citar: Loyacono N, Sanz ML. Gerbi MD, Martínez LM, et al. Problemas gastrointestinales, nutricionales, endocrinológicos y de microbiota en el trastorno del espectro autista. Arch Argent Pediatr 2020;118(3):e271-e277.

\section{INTRODUCCIÓN}

Los trastornos del espectro autista (TEA) se diagnostican sobre la base de alteraciones en la conducta, la comunicación y la interacción recíproca e intereses restringidos. El Centro de Control de Enfermedades (Centers for Disease Control and Prevention, CDC) de EE. UU. reportó, en 2018, que 1 de 59 niños de 8 años de ambos sexos en 11 sitios de seguimiento del CDC en 2014 tenía TEA. ${ }^{1}$

En 2019, se sabe que se encuentran múltiples problemas médicos concomitantes (PMC) en las personas con este diagnóstico. ${ }^{2}$ El modelo genético ve el TEA como síntomas de una encefalopatía estática de base genética prenatal. La neurodiversidad plantea el TEA como forma de ser, como otra manera de estar en el mundo. Esta revisión se enmarca en un nuevo modelo, que lo ve como el nombre dado a síntomas de una encefalopatía crónica, dinámica y sistémica: el enfoque integrador (EI).

Se propone analizar los PMC al diagnóstico (PMCD) de TEA considerando que los tratamientos de estos no deben llamarse tratamientos del TEA. Además, se denominará PMCD contribuyentes, causales o comórbidos a la sintomatología mediante la cual se diagnostica el TEA individual. La palabra concomitantes engloba todos esos roles. Hasta que no se trata (o tratan) adecuadamente, no se sabe si existe correlación o no entre el o los PMCD tratados y la sintomatología bajo la cual el TEA se diagnostica. Este proceso no es rápido, simple o directo. Requiere un análisis exhaustivo del médico experto a cargo, como así también capacitación y actualización continua y compromiso familiar.

\section{METODOLOGÍA}

Se consideraron revisiones, artículos clave o consensos publicados sobre problemas gastrointestinales, 
nutricionales y endocrinológicos en el TEA. Se usaron los buscadores Pubmed, Science Direct y Medline. Se dio prioridad a las publicaciones en revistas internacionales con altos índices de impacto (período 2009-mayo de 2019). Se citaron algunas de años anteriores por su importancia. Los criterios de búsqueda incluyeron palabras clave en inglés con autism o ASD y gastrointestinal, nutrition, microbiota, dysbiosis, endocrine, intestinal permeability o digestive enzymes.

\section{RESULTADOS}

Se presentará, a continuación, el análisis de la literatura específica reciente en los problemas gastrointestinales, de microbiota, nutricionales y endocrinológicos en TEA.

\section{Problemas gastrointestinales}

Seis de los 11 pacientes de Kanner tenían alteraciones con la alimentación o con la dieta. ${ }^{3}$ Por décadas, los problemas gastrointestinales se consideraron alrededor del mundo como parte del autismo. En la Argentina, frente al diagnóstico de TEA, los problemas gastrointestinales no son tenidos en cuenta. Esta actitud debe ser revisada, como se ha reportado. ${ }^{4}$

En 2019, existen múltiples publicaciones que confirman innumerables reportes pasados y actuales de los padres sobre problemas gastrointestinales en TEA. A menudo, pueden relacionarse con conductas problemáticas, respuestas sensoriales alteradas, la desregulación del sueño y la conducta, depresión, ansiedad, agresión e irritabilidad. ${ }^{5-11}$ El comportamiento de externalización y los síntomas de internalización asociados con problemas gastrointestinales son diferentes para distintas edades en los niños con TEA. ${ }^{12}$ Un metaanálisis reciente confirmó una fuerte asociación entre los desórdenes gastrointestinales y el autismo. ${ }^{13}$ Están significativamente relacionados con TEA para los adultos. ${ }^{14}$

En los niños con TEA, se han descrito esofagitis, gastritis, duodenitis y colitis desde hace 20 años. ${ }^{15}$ Horvath, en 1999, alertaba que la esofagitis por reflujo y la malabsorción de disacáridos podían contribuir a los problemas conductuales de los pacientes no verbales con autismo. Horvath (2002) ${ }^{16}$ reportó problemas gastrointestinales en un $80,2 \%$, y Parracho (2005),${ }^{17}$ en un $91,4 \%$ de los niños con TEA. En la serie de Horvath, el $76 \%$ de los niños con TEA presentaba permeabilidad intestinal, y el $85 \%$, alteraciones en la sulfatación hepática. ${ }^{16}$
La esofagitis por reflujo se ha encontrado en 25/36 (el $69 \%$ ); la gastritis crónica, en 15/36 (el 41,7 \%), y la duodenitis crónica, en 25/36 (el $69 \%) .{ }^{16}$ Parracho et al. reportaron que el $43 \%$ de los pacientes con TEA tenían heces anormales. ${ }^{17}$

Kang et al. encontraron que 80 (el $49 \%$ ) de 164 pacientes con TEA tenía, al menos, 1 problema gastrointestinal. ${ }^{18}$ Hasta 3276 (el $52 \%$ ) de 6300 pacientes con TEA mostraron problemas gastrointestinales en el Autism Treatment NetworkAutism Speaks (ATN-AS, EE. UU.). ${ }^{19}$ Schrieber et al., reportaron que el $61 \%$ de los niños con TEA tenían síntomas gastrointestinales correlacionados con síntomas afectivos y conductuales. ${ }^{8}$ Furuta et al. presentaron un algoritmo para abordar la constipación en los niños con TEA..$^{20}$ Peters et al., reportaron que sus resultados sugerían que los clínicos debían evaluar por constipación y diarrea o síntomas relacionados a los niños con TEA que tenían síntomas rígido-compulsivos prominentes. ${ }^{11}$ Ferguson et al., informaron que 221 (el $65 \%$ ) de 340 niños y adolescentes con TEA (2-18 años) mostraban constipación. ${ }^{12}$

La heterogeneidad encontrada en los resultados de tratamientos de PMCD se relaciona con el diseño de los estudios clínicos. Estos no han considerado PMCD adicionales a los gastrointestinales, que pueden afectar el progreso del tratamiento de un PMCD. Así, se ha estudiado el impacto de la dieta en múltiples publicaciones, pero no se han evaluado otros aspectos. El estado de la microbiota, deficiencias nutricionales, alteraciones endocrinológicas y problemas inmunes y autoinmunes pueden estar presentes y afectar los resultados de los estudios. Debe citarse la falta de análisis de laboratorio y las diferencias individuales en la presentación y sintomatología de problemas gastrointestinales en el TEA. Además, existen importantes dificultades para que el médico logre el diagnóstico de estos problemas (sobre todo, en los niños no verbales o con alteraciones en la integración sensorial).

Los hallazgos en TEA más importantes son permeabilidad intestinal aumentada, ${ }^{21}$ diarrea, constipación, reflujo gastroesofágico en todos los grados, alteraciones en las enzimas hepáticas, deficiencias enzimáticas digestivas y disbiosis intestinal. Esta puede incluir alteraciones en la flora bacteriana benigna e infecciones fúngicas, parasitarias y bacterianas. ${ }^{5,6,22,23}$ En muchos casos, estos problemas médicos coexisten con otros, como los mitocondriales, toxicológicos, inmunes y autoinmunes. ${ }^{4}$ 
De Magistris et al. encontraron permeabilidad intestinal en el $36,7 \%$ de los pacientes con TEA versus el $4,8 \%$ de los controles. Los problemas gastrointestinales estuvieron presentes en el $46,7 \%$ de los niños con autismo: constipación (el 45,5\%), diarrea (el 34,1 \%) y otros (diarrea / constipación alternantes, dolor abdominal, etc., en el 15,9\%). Se reportó que los hallazgos en los niños con TEA con síntomas gastrointestinales y colitis apoyaban la presencia de una enfermedad inflamatoria intestinal. ${ }^{21}$ Existe evidencia de alta calidad que indica que los niños más gravemente afectados en TEA son los que presentan síntomas gastrointestinales más graves. Gorrindo et al., confirmaron que la presencia de disfunción gastrointestinal no estaba asociada con distintos hábitos dietarios o el estado de la medicación. El reporte de los padres de las disfunciones gastrointestinales es altamente concordante con el diagnóstico clínico posterior. ${ }^{23}$

Un trabajo de consenso ${ }^{20}$ (igual a los 2 trabajos de consenso publicados en 2010 por Buie et al., en Pediatrics) ${ }^{22,25}$ recomendó que los proveedores de salud debían ser alertados sobre el hecho de que las manifestaciones gastrointestinales en los pacientes con TEA podían ser atípicas y evidentes solo como un cambio en el comportamiento, lo que presentaba un desafío significativo para ambos, padres y médicos. Esto puede incluir la aparición de autoagresión, irritabilidad, agresión o movimientos y posturas extrañas. Kang et al., mencionan que "los síntomas de la disfunción gastrointestinal estuvieron asociados con desórdenes del sueño e intolerancias alimentarias". ${ }^{18}$

Lamentablemente, los problemas gastrointestinales (entre otros PMCD) no son considerados en forma rutinaria en evaluaciones de personas con diagnóstico de TEA. Esto refleja varios factores. La variabilidad en la prevalencia reportada de desórdenes gastrointestinales (en general, sin consideración de la presencia o no de PMCD adicionales) es un problema. Las controversias en relación con los síntomas gastrointestinales y las causas del autismo y la limitada capacidad verbal de muchos pacientes con TEA son otros. Debe considerarse la falta de reconocimiento de los médicos clínicos y médicos pediatras (y otros, como neurólogos y psiquiatras pediátricos) de las manifestaciones conductuales en las personas con TEA como indicadores de problemas gastrointestinales y otros. En general, se las ve como manifestaciones del autismo. Esto refleja actitudes relacionadas con la formación en los modelos aplicados (genético, de la neurodiversidad o psicoanalítico).

\section{Microbiota}

Fattorusso et al. en 2019 confirman que la disbiosis ha sido ampliamente demostrada en TEA y enfatizan el rol de los probióticos. ${ }^{26}$ Sanctuary et al. (2018) confirman que la combinación de ingestas de proteínas específicas, pobre digestión, integridad disminuida de la barrera intestinal y composición y función alteradas de la microbiota representan un patrón fenotípico en TEA. ${ }^{27}$ Se ha publicado la relación entre el sistema inmune y el gastrointestinal. ${ }^{28}$ Se reportó la presencia de múltiples concomitancias en distintos subgrupos de personas con diagnóstico de TEA y déficit de atención con hiperactividad. ${ }^{29}$

Kushak et al. mostraron que la frecuencia de deficiencia de lactasa fue del $58 \%$ en los niños menores de 5 años y del $65 \%$ en los pacientes mayores con autismo. ${ }^{30}$ Sin embargo, no se explora clínicamente por protocolo en la población infantil con diagnóstico de TEA. Williams et al. (2011) demostraron que la transcripción ileal de disacaridasas y transporte de hexosas eran deficientes en el autismo. El análisis metagenómico de las bacterias intestinales reveló disbiosis. Se encontró una disminución en Bacteroidetes, aumento en la relación de Firmicutes a Bacteroidetes e incrementos en Betaproteobacteria. ${ }^{31}$

Los estudios en los pacientes con TEA han revelado la presencia de bacterias anormales que no están presentes en los controles. Se ha encontrado translocación de especies bacterianas a partes del sistema gastrointestinal que no son huéspedes de esas bacterias en individuos sanos. ${ }^{17,31-33}$ Especies desulfovibrio fueron detectadas en pacientes con diagnóstico de TEA, pero no en controles. ${ }^{34}$ Parracho et al., ${ }^{17}$ reportaron que el $34,5 \%$ de los niños con TEA y el $33,3 \%$ de sus hermanos habían recibido más de 6 cursos de antibióticos, usualmente, para desórdenes del tracto respiratorio o infecciones de oído versus el $0 \%$ de un grupo no relacionado control. En su grupo de pacientes con TEA (el 80,2\% con problemas gastrointestinales), el porcentaje alcanzaba el $54,5 \%$. Niehus et al., hallaron un uso mayor de antibióticos en el período de 0 a 2 años en 75 niños con TEA que luego recibieron diagnóstico de autismo en relación con los controles. ${ }^{35}$

Las anormalidades en la flora gastrointestinal en TEA se encuentran confirmadas. ${ }^{36-38}$ Wang et al., brindaron una revisión actualizada sobre 
marcadores en relación con alteraciones de la flora bacteriana. ${ }^{38}$ Kesli et al., publicaron evidencia sobre la infección por Clostridia en autismo. Se encontró una diferencia estadísticamente significativa en el grupo con autismo de ácido 3-(3-hidroxi fenil)-3-hidroxipropiónico (HPHPA) en orina, un metabolito de Clostridia. ${ }^{39}$

Los cambios metabólicos/bioquímicos encontrados confirmaron anormalidades en la microbiota intestinal. ${ }^{40,41}$ Se encontraron toxinas bacterianas en la sangre y se correlacionaron con la gravedad de los síntomas del TEA..$^{37,42} \mathrm{La}$ endotoxemia resultaría de la presencia aumentada de bacterias patógenas más la permeabilidad intestinal incrementada. Un estudio reportó el $36,5 \%$ de inmunoglobulina G (IgG) anti Candida en el plasma, ${ }^{43}$ y otro, un $57,5 \%$ de muestras de materia fecal de niños con TEA con presencia elevada de Candida. ${ }^{44}$

La inflamación crónica e infecciones del tracto intestinal se asocian con un sistema inmune activado y con niveles incrementados de citoquinas proinflamatorias. Esto tiene efecto directo cerebral y repercute en la conducta, que incluye el aumento de la ansiedad, problemas con la motivación y con la socialización, el evitar situaciones nuevas y la adherencia a acciones rutinarias y repetitivas. ${ }^{45-47}$

Existe una nueva subárea, conocida como la psicomicrobiótica. ${ }^{48} \mathrm{El}$ sobrecrecimiento de bacterias y hongos patógenos (disbiosis), la fermentación anormal y la producción de ácidos orgánicos cortos se encuentran, muy a menudo, en el TEA. Las deficiencias enzimáticas de disacaridasas, la permeabilidad intestinal, las parasitosis, las alergias / intolerancias/sensibilidad a proteínas dietarias (como gluten, caseína y otras de la soja) o hidratos de carbono y otras alteraciones forman parte del cuadro de PMCD de TEA y sus efectos. Estos efectos están mediados a través del eje hipotalámico-pituitaria-adrenales, el sistema del nervio vago/sistema nervioso autónomo y la señalización de la serotonina, todos reportados anormales en el autismo. ${ }^{49,50}$

La permeabilidad intestinal en TEA (o leaky $g u t)$, junto con el desbalance en la microbiota, ha sido analizada por Sajdel-Sulkowska et al. ${ }^{51}$ También se ha correlacionado con la disfunción mitocondrial en TEA. ${ }^{52}$ Esto se acompaña con reportes de permeabilidad incrementada de barrera hematocefálica como hallazgo distintivo del TEA. ${ }^{33,54}$

\section{Problemas nutricionales}

Neggers reportó la importancia del ácido fólico (vitamina B9) y la vitamina D materna en el riesgo de autismo..$^{55}$ Por otra parte, los niveles séricos de vitamina $\mathrm{D}$ se midieron en 50 niños con autismo (de 5 a 12 años) y 30 controles. Un $40 \%$ y un $48 \%$ demostraron ser deficientes o insuficientes, respectivamente, en vitamina D. ${ }^{56}$ Trivedi et al. demostraron como los péptidos derivados de gluten y caseína ricos en prolina modulaban el transporte de cisteína (clave en la síntesis del glutatión) en células epiteliales gastrointestinales y neuronales. ${ }^{57} \mathrm{El}$ tratamiento de alergias alimentarias, tomando en cuenta la dieta, se ha reportado en el marco de revisiones bibliográficas recientes, tanto para PMCD de TEA como en déficit de atención con hiperactividad. ${ }^{58}$

Un estudio de casos y controles con 40 niños con TEA y 113 niños sanos de 6 a 10 años mostró que el $20 \%$ tenía índices de masa corporal debajo del percentil 5 para niños de desarrollo típico. ${ }^{59}$ Kawicka et al. (2013), en su revisión, plantearon que la terapia dietaria era insuficiente si se pretendía "tratar autismo". El tratamiento nutricional trataba uno o varios PMCD de TEA debidamente diagnosticados cuyos resultados podían afectar o no la sintomatología del TEA. Los autores demostraron la necesidad de suplir en el caso de deficiencias comprobadas en ácidos grasos omega-3, vitaminas y minerales y probióticos para alteraciones en la flora intestinal, en combinación con intervenciones psicológicas y médicas. ${ }^{60}$

Ali et al. (2011) reportaron altos valores de homocisteína y bajo folato y vitamina B12 en un total de 40 personas con TEA. ${ }^{61}$ Hamza et al. (2013) publicaron que el $54 \%$ de 50 niños con autismo y el $58 \%$ de sus madres eran deficientes en yodo. Esta deficiencia se correlacionó inversamente con la gravedad del TEA. ${ }^{62}$ Tres estudios encontraron niveles bajos de ácidos grasos esenciales y un cuarto no. ${ }^{63-66}$

Adams et al. (2011) mostraron que 55 niños con autismo, en relación con 44 controles, presentaban diferencias significativas, debido a la insuficiencia de vitaminas del complejo B, entre otras. Los autores encontraron estrés oxidativo incrementado, reducida capacidad para transporte de energía y alterada sulfatación y desintoxicación. ${ }^{67}$

Pagan et al. estudiaron el nivel de serotonemia y de melatoninemia en un grupo de 278 pacientes con TEA, 506 parientes de primer grado y 416 controles sanos. Con el $40 \%$ (el 35-46 \%) de 
los pacientes con TEA encontraron valores de serotonina superiores al percentilo 95 de los valores y en el $51 \%$ (el 47-57 \%) los valores de melatonina eran inferiores al percentilo 5 de los otros grupos. ${ }^{68}$

Una revisión de 2013 sobre el estado nutricional y las preferencias alimentarias en TEA $^{60}$ estableció que, además de desarrollar obesidad, los pacientes con TEA demostraban riesgo de malnutrición debido a la ingesta inadecuada de energía de la dieta y a los problemas causados por la absorción inadecuada intestinal de nutrientes aparte de indigestión. Cualquier desviación de un estado nutricional normal (sobrepeso, obesidad, caquexia) en pacientes con TEA debería ser una señal para promover un tratamiento dietario.

\section{Problemas endocrinológicos}

Tareen et al. encontraron T3 baja con estrés oxidativo y disminución de la actividad D2 en TEA. ${ }^{69}$ Sin embargo, los resultados de las medidas de hormonas tiroideas neonatales fueron normales para 78 pacientes. ${ }^{70}$ Desoky et al. reportaron valores significativamente mayores de la hormona estimulante de la tiroides (thyroid-stimulating hormone; TSH, por sus siglas en inglés), con elevada expresión de CD5 y valores disminuidos de vitamina $\mathrm{D}$ en TEA. ${ }^{71}$ Se han reportado niveles significativamente más bajos de colesterol total en suero, cortisol y estradiol, así como dehidroepiandrostenediona y testosterona libre mayores en varones con TEA. ${ }^{72}$ Los esteroides desregulados pueden afectar la ansiedad, generar perturbaciones en el sueño, déficits sensoriales y estereotipias.

Las personas con TEA tienen mayor prevalencia de problemas médicos, hasta 9 veces mayor mortalidad que la población típica, mayor utilización del sistema de salud y con mayores costos. Sin embargo, experimentan múltiples barreras en lograr el cuidado médico apropiado en EE. UU. ${ }^{73}$ En la Argentina, la situación es similar. Esto debería revertirse. Existe bibliografía local que reporta algoritmos de evaluación de PMCD del TEA. ${ }^{4}$ Adams et al. en 2018 publicaron un estudio clínico aleatorizado, controlado, simple ciego de 12 meses de una intervención nutricional y dietética integral con 67 niños y adultos con TEA de 3 a 58 años. Se reportó un aumento en el coeficiente intelectual no verbal y en la comunicación, habilidades de la vida diaria y habilidades sociales. Se encontró una reducción en los comportamientos aberrantes, dificultades sensoriales y síntomas gastrointestinales y del autismo. $^{74}$

Los genes encontrados asociados a TEA podrían aumentar la susceptibilidad para contraer PMCD extracerebrales. Se han encontrado correlaciones con el sistema inmune y distintas rutas metabólicas. ${ }^{2}$ La literatura reciente insiste en la necesidad de evaluar, diagnosticar y tratar PMCD para optimizar la salud, el progreso y la calidad de vida en el marco de los apoyos necesarios para la sintomatología externa en TEA..$^{4,75,76}$ Es importante recalcar que la prevalencia se ha reportado entre el $46 \%$ y el $89 \%$ para los problemas nutricionales, ${ }^{77}$ más del $50 \%$ para la disfunción tiroidea ${ }^{78}$ y hasta el $100 \%$ para las alteraciones en la microbiota. ${ }^{36,45,51,52}$

Los profesionales de la salud deberían considerar la disfunción gastrointestinal (y otros PMCD) en todo paciente con TEA, pero, en particular, en aquellos que presentan movimientos o posturas extrañas, desórdenes del sueño, déficit de atención, hiperactividad, intolerancias o selectividad alimentarias, comportamientos asociables a reacciones adversas a los alimentos y comportamientos agresivos y autoagresivos.

\section{CONCLUSIONES}

Este artículo muestra parte de la base bibliográfica para la consideración personalizada de PMCD del TEA en los pacientes pediátricos, jóvenes y adultos. Expone la importancia del diagnóstico y tratamiento correcto de los PMCD, el tiempo necesario para brindar calidad de vida para el paciente y su familia, y enfatiza la necesidad de considerar los aportes de los padres en forma cuidadosa en cuanto a la historia clínica y de formar un equipo con la familia. Se debería brindar información actualizada a 2019 de alta calidad sobre PMCD del TEA, modelos avanzados de abordaje y la importancia del compromiso familiar con el tratamiento de los PMCD del TEA.

\section{REFERENCIAS}

1. Baio J, Wiggins L, Christensen DL, Maenner MJ, et al. Prevalence of Autism Spectrum Disorder Among Children Aged 8 Years - Autism and Developmental Disabilities Monitoring Network, 11Sites, United States, 2014. MMWR Surveill Summ. 2018; 67(6):1-23.

2. Tye C, Runicles AK, Whitehouse AJO, Alvares GA. Characterizing the Interplay Between Autism Spectrum Disorder and Comorbid Medical Conditions: An Integrative Review. Front Psychiatry. 2019; 9:751.

3. Kanner L. Autistic disturbances of affective contact. Nervous Child. 1943; 2:217-50. 
4. Loyacono N, Ferreira ML, Iermoli R. Humanismo en medicina. El rol crucial del pediatra en el trastorno del espectro autista. Arch Argent Pediatr. 2019; 117(3):195-7.

5. Holingue C, Newill C, Lee LC, Pasricha PJ, et al. Gastrointestinal symptoms in autism spectrum disorder: A review of the literature on ascertainment and prevalence. Autism Res. 2018; 11(1):24-36.

6. Chaidez V, Hansen RL, Hertz-Picciotto I. Gastrointestinal problems in children with autism, developmental delays or typical development. J Autism Dev Disord. 2014; 44(5): 1117-27.

7. Chandler S, Carcani-Rathwell I, Charman T, Pickles A, et al. Parent-reported gastro-intestinal symptoms in children with autism spectrum disorders. J Autism Dev Disord. 2013; 43(12):2737-47.

8. Mazefsky CA, Schreiber DR, Olino TM, Minshew NJ. The association between emotional and behavioral problems and gastrointestinal symptoms among children with highfunctioning autism. Autism. 2014; 18(5):493-501.

9. Coury DL, Ashwood P, Fasano A, Fuchs G, et al. Gastrointestinal conditions in children with autism spectrum disorder: developing a research agenda. Pediatrics. 2012; 130(Suppl 2):S160-8

10. Mazurek MO, Vasa RA, Kalb LG, KanneSM, et al. Anxiety, sensory over-responsivity, and gastrointestinal problems in children with autism spectrum disorders. J Abnorm Child Psychol. 2013; 41(1):165-76.

11. Peters B, Williams KC, Gorrindo P, Rosenberg D, et al. Rigid-Compulsive Behaviors are Associated with Mixed Bowel Symptoms in Autism Spectrum Disorder. J Autism Dev Disord. 2014; 44(6):1425-32.

12. Ferguson BJ, Dovgan K, Takahashi N, Beversdorf DQ. The Relationship Among Gastrointestinal Symptoms, Problem Behaviors, and Internalizing Symptoms in Children and Adolescents With Autism Spectrum Disorder. Front Psychiatry. 2019; 10:194.

13. McElhanon BO, McCracken C, Karpen S, Sharp WG. Gastrointestinal Symptoms in Autism Spectrum Disorder: A Meta-analysis. Pediatrics. 2014; 133(5):872-83.

14. Croen LA, Zerbo O, Qian Y, Massolo ML, et al. The health status of adults on the autism spectrum. Autism. 2015; 19(7):814-23.

15. Horvath K, Papadimitriou JC, Rabsztyn A, Drachenberg C, et al. Gastrointestinal abnormalities in children with autistic disorder. J Pediatr. 1999; 135(5):559-63.

16. HorvathK,PermanJA.Autistic disorderand gastrointestinal disease. Curr Opin Pediatr. 2002; 14(5):583-7.

17. Parracho HM, Bingham MO, Gibson GR, McCartney AL. Differences between the gut microflora of children with autistic spectrum disorders and that of healthy children. $J$ Med Microbiol. 2005; 54(Pt 10):987-91.

18. Kang V, Wagner GC, Ming X. Gastrointestinal dysfunction in children with autism spectrum disorders. Autism Res. 2014; 7(4):501-6.

19. Perrin J. Autism spectrum disorder and comorbid conditions. In: Interagency Autism Coordinating Committee. Bethesda, Maryland: July 9, 2013. [Acceso: 26 de diciembre de 2019]. Disponibleen:https: / / iacc.hhs.gov / meetings / iacc-meetings / 2013/ full-committee-meeting/ july9/slides_james_perrin_070913b.pdf.

20. Furuta GT, Williams K, Kooros K, Kaul A, etal. Management of Constipation in Children and Adolescents With Autism Spectrum Disorders. Pediatrics. 2012; 130(Suppl2);S98-105.

21. De Magistris L, Familiari V, Pascotto A, Sapone A, et al. Alterations of the intestinal barrier in patients with autism spectrum disorders and in their first-degree relatives. $J$ Pediatr Gastroenterol Nutr. 2010; 51(4):418-24.

22. Buie T, Campbell DB, Fuchs GJ 3rd, Furuta GT, et al.
Evaluation, diagnosis, and treatment of gastrointestinal disorders in individuals with ASDs: a consensus report. Pediatrics. 2010; 125(Suppl 1):S1-18.

23. Gorrindo P, Williams KC, Lee EB, Walker LS, et al. Gastrointestinal dysfunction in autism: parental report, clinical evaluation, and associated factors. Autism Res. 2012; 5(2):101-8.

24. Walker SJ, Fortunato J, Gonzalez LG, Krigsman A. Identification of unique gene expression profile in children with regressive autism spectrum disorder (ASD) and ileocolitis. PLoS One. 2013; 8(3):e58058.

25. Buie T, Fuchs GJ 3rd, Furuta GT, Kooros K, et al. Recommendations for evaluation and treatment of common gastrointestinal problems in children with ASDs. Pediatrics. 2010; 125(Suppl 1):S19-29.

26. Fattorusso A, Di Genova L, Dell'Isola GB, Mencaroni E, et al. Autism Spectrum Disorders and the Gut Microbiota. Nutrients. 2019; 11(3):E521.

27. Sanctuary MR, Kain JN, Angkustsiri K, German JB. Dietary Considerations in Autism Spectrum Disorders: The Potential Role of Protein Digestion and Microbial Putrefaction in the Gut-Brain Axis. Front Nutr. 2018; 5:40.

28. Rudzki L, Szulc A. "Immune Gate" of PsychopathologyThe Role of Gut Derived Immune Activation in Major Psychiatric Disorders. Front Psychiatry. 2018; 9:205.

29. Muskens JB, Velders FP, Staal WG. Medical comorbidities in children and adolescents with autism spectrum disorders and attention deficit hyperactivity disorders: a systematic review. Eur Child Adolesc Psychiatry. 2017; 26(9):1093-103.

30. Kushak RI, Lauwers GY, Winter HS, Buie TM. Intestinal disaccharidase activity in patients with autism: effect of age, gender, and intestinal inflammation. Autism. 2013; 15(3):285-94.

31. Williams BL, Hornig M, Buie T, Bauman ML, et al. Impaired carbohydrate digestion and transport and mucosal dysbiosis in the intestines of children with autism and gastrointestinal disturbances. PLoS One. 2011; 6(9):e24585.

32. De Angelis M, Piccolo M, Vannini L, Siragusa S, et al. Fecal microbiota and metabolome of children with autism and pervasive developmental disorder not otherwise specified. PLoS One. 2013; 8(10):e76993.

33. Ekiel A, Aptekorz M, Kazek B, Wiechula B, et al. Intestinal microflora of autistic children. Med Dosw Mikrobiol. 2010; 62(3):237-43

34. Finegold SM, Dowd SE, Gontcharova V, Liu C, et al. Pyrosequencing study of fecal microflora of autistic and control children. Anaerobe. 2010; 16(4):444-53.

35. Niehus R, Lord C. Early medical history of children with autism spectrum disorders. J Dev Behav Pediatr. 2006; 27(2 Suppl):S120-7.

36. Cao X, Lin P, Jiang P, Li C. Characteristics of the gastrointestinal microbiome in children with autism spectrum disorder: a systematic review. Shanghai Arch Psychiatry. 2013; 25(6):342-53.

37. Hsiao EY. Gastrointestinal issues in autism spectrum disorder. Harv Rev Psychiatry. 2014; 22(2):104-11.

38. Wang L, Christophersen CT, Sorich MJ, Gerber JP, et al. Increased abundance of Sutterella spp. and Ruminococcus torques in feces of children with autism spectrum disorder. Mol Autism. 2013; 4(1):42.

39. Keşli R, Gökçen C, Buluğ U, Terzi Y. Investigation of the relation between anaerobic bacteria genus clostridium and late-onset autism etiology in children. J Immunoassay Immunochem. 2014; 35(1):101-9.

40. Ming X, Stein TP, Barnes V, Rhodes N, et al. Metabolic perturbance in autism spectrum disorders: A metabolomics study. J Proteome Res. 2012; 11(12):5856-62.

41. Yap IK, Angley M, Veselkov KA, Holmes E, et al. Urinary 
metabolic phenotyping differentiates children with autism from their unaffected siblings and age-matched controls. J Proteome Res. 2010; 9(6):2996-3004.

42. Emanuele E, Orsi P, Boso M, Broglia D, et al. Low-grade endotoxemia in patients with severe autism. Neurosci Lett. 2010;471(3):162-5.

43. Hughes HK, Ashwood P. Anti-Candida albicans IgG Antibodies in Children With Autism Spectrum Disorders. Front Psychiatry. 2018; 9:627.

44. IoveneMR, Bombace F, Maresca R, Sapone A, et al. Intestinal Dysbiosis and Yeast Isolation in Stool of Subjects with Autism Spectrum Disorders. Mycopathologia. 2017; 182(34):349-63.

45. Cryan JF, Dinan TG. Mind-altering microorganisms: the impact of the gut microbiota on brain and behaviour. Nat Rev Neurosci. 2010; 13(10):701-12.

46. Goehler LE, Gaykema RP. Neural pathways mediating behavioral changes associated with immunological challenge. In: Siegel A, Zalcman S (eds.). The Neuroimmunological Basis of Behavior and Mental Disorders. New York: Springer; 2009.Págs.35-58.

47. Goehler LE, Gaykema R, Opitz N, Reddaway R, et al. Activation in vagal afferents and central autonomic pathways: Early responses to intestinal infection with Campylobacter jejuni. Brain Behav Immun. 2005; 19(4):33444.

48. Fond G, Boukouaci W, Chevalier G, Regnault A, et al. The "psychomicrobiotic": Targeting microbiota in major psychiatric disorders: A systematic review. Pathol Biol (Paris). 2015; 63(1):35-42.

49. Diaz Heijtz R, Wang S, Anuar F, Qian Y, et al. Normal gut microbiota modulates brain development and behavior. Proc Natl Acad Sci U S A. 2011; 108(7):3047-52.

50. Foster JA, McVey Neufeld KA. Gut-brain axis: how the microbiome influences anxiety and depression. Trends Neurosci. 2013; 36(5):305-12.

51. Sajdel-Sulkowska EM, Zabielski R. Determinans of individual sensitivity of brain-gut axis and gut microbiome to enviromental toxins; intrinsic and extrinsic components. In: Gut Microbiome and Brain-Gut Axis in Autism Aberrant Development of Gut-Brain Communication and Reward Circuitry. Recent Advances in Autism Spectrum Disorders - 2013. [Acceso: 20 de diciembre de 2019]. Disponible en: https://www.intechopen.com/books / recent-advances-in-autism-spectrum-disorders-volume-i / gut-microbiome-and-brain-gut-axis-in-autism-aberrantdevelopment-of-gut-brain-communication-and-rewa.

52. Macfabe D. Autism: metabolism, mitochondria, and the microbiome. Glob Adv Health Med. 2013; 2(6):52-66.

53. Kealy J, Greene C, Campbell M. Blood-brain barrier regulation in psychiatric disorders. Neurosci Lett. 2018; 30:133664.

54. Fiorentino M, Sapone A, Senger S, Camhi SS, et al. Bloodbrain barrier and intestinal epithelial barrier alterations in autism spectrum disorders. Mol Autism. 2016; 7:49.

55. Neggers YH. Increasing prevalence, changes in diagnostic criteria, and nutritional risk factors for autism spectrum disorders. ISRN Nutr. 2014; 2014:514026.

56. Mostafa GA, Al-Ayadhi LY. Reduced serum concentrations of 25-hydroxy vitamin $\mathrm{D}$ in children with autism: relation to autoimmunity. J Neuroinflammation. 2012; 9:201.

57. Trivedi MS, Shah JS, Al-Mughairy S, Hodgson NW, et al. Food-derived opioid peptides inhibit cysteine uptake with redox and epigenetic consequences. J Nutr Biochem. 2014; 25(10):1011-8.

58. De Theije CG, Bavelaar BM, Lopes da Silva S, Korte $\mathrm{SM}$, et al. Food allergy and food-based therapies in neurodevelopmental disorders. Pediatr Allergy Immunol. 2014; 25(3):218-26.

59. Bauset SM, Zazpe I, Sanchis AM, González AL, et al. Are there anthropometric differences between autistic and healthy children? J Child Neurol. 2013; 28(10):1226-32.
60. Kawicka A, Regulska-Ilow B. How nutritional status, diet and dietary supplements can affect autism. A review. Rocz Panstw Zakl Hig. 2013; 64(1):1-12.

61. Ali A, Waly MI, Al-Farsi YM, Essa MM, et al. Hyperhomocysteinemia among Omani autistic children: a case-control study. Acta Biochim Pol. 2011; 58(4):547-51.

62. Hamza RT, Hewedi DH, Sallam MT. Iodine deficiency in Egyptian autistic children and their mothers: relation to disease severity. Arch Med Res. 2013; 44(7):555-61.

63. Bell JG, MacKinlay EE, Dick JR, MacDonald DJ, et al. Essential fatty acids and phospholipase A2 in autistic spectrum disorders. Prostaglandins Leukot Essent Fatty Acids. 2004; 71(4):201-4.

64. Meguid NA, Atta HM, Gouda AS, Khalil RO. Role of polyunsaturated fatty acids in the management of Egyptian children with autism. Clin Biochem. 2008; 41(13):1044-8.

65. Vancassel S, Durand G, Barthelemy C, Lejeune B, et al. Plasma fatty acid levels in autistic children. Prostaglandins Leukot Essent Fatty Acids. 2001; 65(1):1-7.

66. Bu B, Ashwood P, Harvey D, King IB, et al. Fatty acid compositions of red blood cell phospholipids in children with autism. Prostaglandins Leukot Essent Fatty Acids. 2006; 74(4):215-21.

67. Adams JB, Audhya T, McDonough-Means S, Rubin RA, et al. Effect of a vitamin/mineral supplement on children and adults with autism. BMC Pediatr. 2011; 11:111.

68. Pagan C, Delorme R, Callebert J, Goubran-Botros H, et al. The serotonin-N-acetylserotonin-melatonin pathway as a biomarker for autism spectrum disorders. Transl Psychiatry. 2014; 4:e479.

69. Tareen RS, Kamboj MK. Role of endocrine factors in autistic spectrum disorders. Pediatr Clin North Am. 2012; 59(1):75-88.

70. Yau VM, Lutsky M, Yoshida CK, Lasley B, et al. Prenatal and Neonatal Thyroid Stimulating Hormone Levels and Autism Spectrum Disorders. J Autism Dev Disord. 2015; 45(3):719-30.

71. Desoky T, Hassan MH, Fayed HM, Sakhr HM. Biochemical assessments of thyroid profile, serum 25-hydroxycholecalciferol and cluster of differentiation 5 expression levels among children with autism. Neuropsychiatr Dis Treat. 2017; 13:2397-403.

72. Hassan MH, Desoky T, Sakhr HM, Gabra RH, et al. Possible Metabolic Alterations among Autistic Male Children: Clinical and Biochemical Approaches. J Mol Neurosci. 2019; 67(2):204-16.

73. Tregnago MK, Cheak-Zamora NC. Systematic review of disparities in health care for individuals with autism spectrum disorders in the United States. Res Autism Spectr Disord. 2012; 6(3):1023-31.

74. Adams JB, Audhya T, Geis E, Gehn E, et al. Comprehensive Nutritional and Dietary Intervention for Autism Spectrum Disorder-A Randomized, Controlled 12-Month Trial. Nutrients. 2018; 10(3):E369.

75. Frye RE, Rossignol DA. Identification and Treatment of Pathophysiological Comorbidities of Autism Spectrum Disorder to Achieve Optimal Outcomes. Clin Med Insights Pediatr. 2016; 10:43-56.

76. Loyacono N, Ferreira ML, Iermoli RH. Trastornos del Espectro Autista. EnfoqueIntegrador: del arte delo posible a la Investigación puesta en Práctica. Buenos Aires: Ediciones Globales; 2018.

77. Ledford JR, Gast DL. Feeding Problems in Children With Autism Spectrum Disorders A Review. Focus Autism Other Dev Disabl. 2006; 21(3):153-66.

78. Frye RE, Wynne R, Rose S, Slattery J, et al. Thyroid dysfunction in children with autism spectrum disorder is associated with folate receptor $\alpha$ autoimmune disorder. J Neuroendocrinol. 2017;29(3). doi: 10.1111/jne.12461. 